\title{
Design of Hybrid Method for Automatic Sentiment Classification System
}

\author{
D. Aruna Kumari, P. Rajashekar, PKVS Sarma
}

\begin{abstract}
The Developing enthusiasm for the field of opinion mining and its applications in various regions of information and also, sociology has activated numerous researchers to investigate the field The chance to catch the opinion of the overall public about get-togethers, political developments, organization systems, advertising efforts, and item inclinations has raised expanding enthusiasm of both scientific community (as a result of the energizing open difficulties) and the business world (due to the wonderful advantages for promoting and money related market expectation). Today, sentiment analysis investigation has its applications in a few unique situations. There are a decent number of organizations, both huge and little scale, that focuses on opinions and sentiments as a major aspect of their central goal. This work introduces hybrid approach that includes lexicon based approach and machine learning approach for extracting aspects and sentiments
\end{abstract}

keywords: CNN, Sentiment, feature extraction

\section{INTRODUCTION}

Opinion Mining or Sentiment analysis includes the working of a framework that investigates the client conclusions that are made in blog entries, remarks, surveys or tweets about an item or a theme. The mental condition of a client identified with a theme is resolved. Opinion Mining is a subfield of information mining. It is utilized to judge the sentiments of the human kind on the web through reviews. Natural Language processing of massive data sets, including Sentiment examination and sentiment mining has been critical to comprehend customer conduct. It has been demonstrated patient remarks about particular Doctors could have positive or negative sentiment. It is additionally proposed that catching of feelings ought to be identified with regular techniques for assessing quiet encounter. The Information Strategy for the National health Service (NHS) in England expresses that notion investigation of information is the underlying wellspring of significant data for patients in helping to choose hospitals. Scientists have primarily considered sentiment analysis at three levels of granularity: document level, sentence level, and aspect level. Document level characterization orders an opinionated document (e.g., an item survey) as communicating a general positive or negative feeling[1,2]. It thinks about the entire document as the essential data unit and expect that the report is known to be stubborn. Its main task to provide Sentiment Classification of whole document i.e Positive, negative and neutral essential data unit and expect that the report is known to be stubborn. Its main task to provide Sentiment Classification of whole document i.e Positive, negative and neutral Sentence level

Revised Version Manuscript Received on 16 September, 2019.

* Correspondence Author

Dr Aruna Kumari Professor at Department of CSE,VJIT, Hyderabad, Telangana India.. Email: arunakumari@vjit.ac.in

P.Rajashekar, Asst.Professor at Department OF CSE, VJIT ,Hyderabad, Telangana India. Email: rajashekarcse2019@vjit.ac.in

PKVS Sarma, Asst.Professor at Department OF CSE, VJIT, Hyderabad, Telangana India Email: sarma@vjit.ac.in conclusion grouping arranges singular sentences in an archive. Be that as it may, each sentence can't be thought to be determined. Generally, one frequently first characterizes a sentence as obstinate or not stubborn, which is called subjectivity arrangement. At that point the subsequent stubborn sentences are named communicating positive or negative sentiments. Sentence level opinion characterization can likewise be defined as a three-class grouping issue, that is, to arrange a sentence as nonpartisan, positive or negative.

Aspect level deals with labeling each word with their sentiment and also identifying the entity towards which the sentiment is directed. Aspect or Feature level sentiment classification concerns with identifying and extracting product features from the source data.

Various Tasks involved in aspect level mining is

1.Identify and extract object features that have been commented on by an opinion holder (eg. A reviewer)

2.Determining whether the opinions on features are negative, positive or neutral

3.Find feature synonyms

\section{RELATED WORK}

Sentiment classification is a difficult task and a lot of research has been done in the past.. Anna JurekEmail author,Maurice D. Mulvenna and Yaxin Bi have worked on Improved lexicon-based sentiment analysis for social media analytics. ZiedKechaou, Mohamed Ben Ammar, Adel. M Alimi, working on "Improving e-learning with sentiment analysis of users' opinions". Dan Song, Hongfei Lin, Zhihao Yang, published a paper on "Opinion Mining in e- Learning System”, 2007 IFIP International Conference onNetwork and Parallel Computing - Workshop. Stefano Baccianella, Andrea Esuli, and FabrizioSebastiani, reviewed on "SENTI WORD NET 3.0 in 2008. Sandeep Sricharan Mukku, Nurendra Choudhary, Radhika Mamidi from IIT Hyderabad, They worked on "Enhanced Sentiment Classification of Telugu Text using ML Techniques" Another scientist Narayanan et al., 2013 worked on Enhanced Naive Bayes model is used for sentiment classification task in English.Reddy Naidu,Santosh Kumar Bharti, Korra Sathya Babu, Ramesh Kumar Mohapatra from NIT Rourkela, have emphasized more on Sentiment Analysis using Telugu SentiWordNet. Das and Bandyopadhyay [9] deployed a computational technique on English sentiment lexicons and English-Bengali bilingual dictionary to develop a Bengali SentiWordNet.AdityaBhardwaj, YogendraNarayan, Vanraj, Pawan and ..MaitreyeeDuttaworked on Indian Stock market prediction. Traditional approaches frequently use the Bag Of Words (BOW) model, where a document is mapped to a feature vector, and then classified by machine learning techniques. Although the BOW approach is simple and quite efficient, a great deal of the

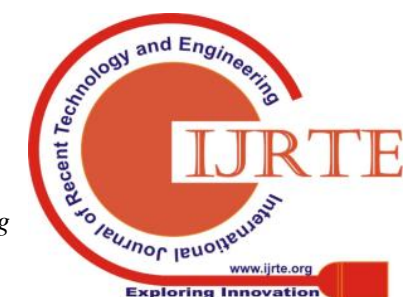


information from the original natural language is lost (Xia \& Zong, 2010), e.g., word order is disrupted and syntactic structures are broken. Therefore, various types of features have been exploited, such as higher order $n$-grams (Pak \& Paroubek, 2010). Another kind of feature that can be used is Part Of Speech (POS) tagging, which is commonly used during a syntactic analysis process, as described in Gimpel et al. (2011). Some authors refer to this kind of features as surface forms, as they consist in lexical and syntactical information that relies on the pattern of the text, rather than on its semantic aspect.

The dominant approaches in sentiment analysis are based on machine learning techniques. Some prior information about sentiment can also be used in the analysis. For instance, by adding individual word polarity to the previously described features (Pablos, Cuadros, \& Rigau, 2016). This prior knowledge usually takes the form of sentiment lexicons, which have to be gathered. Sentiment lexicons are used as a source of subjective sentiment knowledge, where this knowledge is added to the previously described features (Cambria, 2016, Kiritchenko, Zhu, Mohammad, 2014, Melville, Gryc, Lawrence, 2009, Nasukawa, Yi, 2003).

The use of lexicon-based techniques has a number of advantages (Taboada, Brooke, Tofiloski, Voll, \& Stede, 2011). First, the linguistic content can be taken into account through mechanisms such as sentiment valence shifting (Polanyi \& Zaenen, 2006) considering both intensifiers (e.g. very bad) and negations (e.g. not happy). In addition, sentiment orientation of lexical entities can be differentiated based on their characteristics. Moreover, language-dependent characteristics can be included in these approaches

Lexicon Based Sentiment Analysis associate with the presence of certain word in document. Lexicon contains different features including the part of speech tagging of word, their sentiment values, subjectivity of word etc. The Sentiment Analysis of user reviewsare annotate using this features provided by these lexicons. Using that we can obtain polarity of whole reviews by averaging the sentiment values of words. The Machine Learning based Sentiment Analysis technique requires creating a model by training the classifier with labeled examples. This means that first we require to gather a dataset with positive, negative and neutral classes, extract the features/words from that dataset \& then train the algorithm based on the examples. Proposed approach summarize all the customer reviews. Hence, this research work helps to the Users in analyzing the positivity and negativitywith the help of Predictive analytics on collected reviews. Opinions in the form of reviews, extracted from websites and social media will go through automatic sentiment analysis to identify the sentiment of opinions

\section{METHODOLOGY}

Sentiment classification aims to allocate a classification/class to an information or report from a predefined set of classifications/ classes. The predefined classification/classes set for the most part made up of some estimation classes, e.g. "positive" or 'negative'. In supervised machine learning, a prepared factual classifier is utilized for slant order. The prepared classifier extends the introduction of feeling in view of information records. In this manner the machine learning calculations can't work straightforwardly with the information records. Testing of calculation is vital in the event of regulated machine learning calculation. That is the reason a preparatory stage, likewise called pre-processing, is required.

Firstly, textual data should be pre-processed prior classifying it. Pre-processing transforms document data into a relevant format which will make it to the classification phase. The pre-treated documents, go through the phase of removal of punctuation, numbers, stop words and finally stemming. Both training and test sets of documents are pre-processed in the same way.

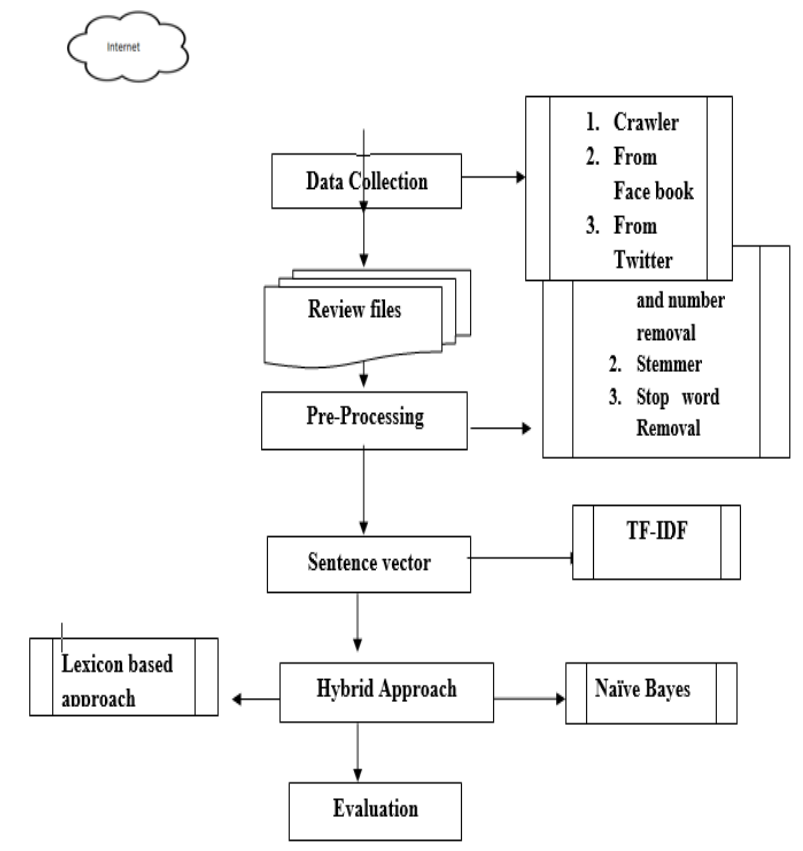

Figure 1 :Aspect based Sentiment analysis

3.1.1 Data Collection :

For Opinion mining on Hospitals, information as surveys are gathered from Social Sites, Twitter what's more, Facebook. The destinations where individuals submit audits of schools like www.Shiksha.com, www.career360.com, the audits are extricated. The extraction of these surveys require plan of crawler particular to the these sites, which will get particular survey for the required healing center. For extraction of audits from Twitter and Facebook, the particular APIs are utilized. The stock of seed URLs helps any Internet crawler. The essential recipe takes it as info and executes the ensuing strides in circle. For the most part an address from the address list is extricated and checked against the science address of its host name. Exchange of the comparing archive and extraction of connections are performed [19]. For every single of the removed connections its Associate outright address is confirmed. Later it is added to the rundown of URLs to get exchanged and gave. .

Pre Processing :

Unwanted symbols, words, numbers must be expelled from gathered audits as these things are not helpful in the procedure of Sentiment Analysis. Stemmer can be utilized for conveying the words to the root shape. Stopword evacuation can be accomplished utilizing the rundown of stopwords.

Punctuations like ",; : " \{ \}) (" and numbers, if display in information after pre-preparing , they may decrease the precision of classifier. So this progression is critical in 
information pre-preparing. Subsequent to gathering last information, a few undesirable accentuations and numbers are expelled from each sentence in informational collection. Content mining bundle in "R" called "tm" [4] evacuates undesirable numbers and Punctuations shape content.

Stemming $[1,3]$ is the term utilized as a part of data recovery to portray the procedure for decreasing bent (or in some cases inferred) words to their root frame. The stems are favored over words serves two valuable part in assumption examination. In the first place, the meager condition in the information is diminished, since there are less particular stems thought about to particular words. Second, semantic data caught and accumulated betterly. For instance, words like "Instructing", "Understanding", and so on., in the wake of stemming is changed over into "educate", "Comprehend", and so on. Thus, with the utilization of stem (root) word, sentence words are pleasantly contrasted and number of positive/negative words making it simple for investigation. Stop words [1, 3], by and large known to be clamor words or most normal words, which don't illuminate any huge reason in the field of feeling arrangement.

Stop words are the most widely recognized words in an any dialect, however there is no particular, single, unequivocal all inclusive rundown of them. They are utilized by all characteristic dialect preparing instruments, and in fact not all apparatuses even utilize such a rundown. With this specific circumstance, we have set up a rundown of words containing mostly English pronouns, particles, extraordinary characters and numbers e.g. relational words, pronouns, furthermore, a few verb modifiers. For instance "The Hospital is great" is a positive sentiment sentence. In the wake of performing the progression of stop words evacuation, the yield inferred is as "Hospital great". This is a proficient and simpler approach to recognize assumption bearing words and elements for ordering the record.

\subsubsection{Sentence vectors :}

The Textual data is represented to numerical arrangement for calculation. Calculation of TF-IDF estimation of every single component (assessment bearing words) from the arrangement of preparing archives is performed. TF- IDF is a standout amongst the most broadly utilized portrayals. TF-IDF strategies consider both term frequencies in a report and in addition the pertinence of a term in the whole gathering of reports. The recipe for ascertaining the TF-IDF can be composed as takes after:

$$
T F=\log (f(t, d)+1)
$$

TF stand for term frequency i.e, number of times the term appears in the document; IDF is the Inversed Document Frequency given by below equation:

$$
I D F=\log (N / n)
$$

' $\mathrm{N}$ ' is the total number of training documents and ' $\mathrm{n}$ ' is the number of documents the term appears. IDF is usefull in minimizing the weight of term with low discriminative value. Hybrid approach (Sentiment Lexicon generation \& Naïve Bayes classifier ) :

Sentiment lexicon generation can be divided into three approaches, namely manual approach, dictionary-based approach, and corpus-based approach. The first approach is built manually by human and thus requires considerable resources. The second approach is dictionary-based approach, where a set of seed words is created manually and then expanded by using a dictionary (thesaurus, WordNet, etc). The corpus-based approach also uses manually labeled seed words and then expanded using available corpus data.

This work uses Dictionary based approach and Naïve bayesclassifer to select the attribute. The Approaches to sentiment analysis using dictionaries such as Senticnet, SentiFul, SentiWordNet, and WordNet are studied in this work. Dictionary- based approaches are efficient over a domain of study

This work is uses a hybrid approach by adding sentiment-bearing words from SentiWordNet 3.0 as features to an Naïve bayesclassifer, beside syntactic and stylistic features. Applies Information Gain heuristic as a feature selection method.

Subjectivity detection \& polarity classification: ㅁ It can’t be done with just a set of subjective keywords! Because it is Context-sensitive. For example, if we take a product review

- This camera is great. (+ve)

- A great amount of money was spent for promoting this camera. (neutral) If you think this is a great camera, well think again, because ... (-ve)

- This film should be brilliant. It sounds like a great plot, the actors are first grade, and the supporting cast is good as well, and Stallone is attempting to deliver a good performance. However, it can't hold up. (-ve).

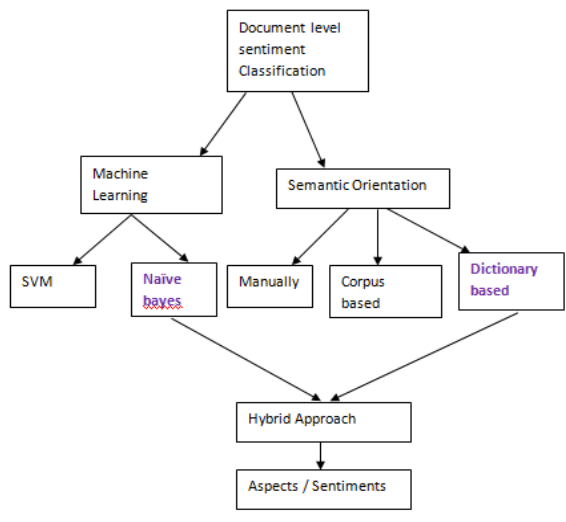

Fig 2: Hybrid approach

REVIEW (i). SentiWordNet for Sentiment Analysis SentiWordNet gives the emotion data to each and every word synset.

We can represent SentiWordNet as Wordnet + sentiment information.This

The words in each file are categorized into five parts-of-speech tags namely, adjective (a), noun (n), adverb $(\mathrm{r})$, verb (v) and unknown (u). We have used neutral words file for the subjectivity classification, negative and positive words file for the sentiment classification. 


\begin{tabular}{|l|l|l|l|}
\hline & Negative & Positive & Neutral \\
\hline Adjective & & & \\
\hline Noun & & & \\
\hline Verb & & & \\
\hline Adverb & & & \\
\hline Unknown & & & \\
\hline
\end{tabular}

Table1: SentiWordNet for Data categorization

Sentiment classifier : Corpus of English words will be considered as input WordNet neutral keywords file (neukf). each word in the sentence will be compared with words in the SentiWordNet positive keyword file (poskf) and negative keyword file (negkf).

If the word is present in poskf, the sentiment of that sentence is considered as positive, and if the word is present in negkf, the sentiment of that sentence is considered as negative. Otherwise, the sentence is simply discarded as any word of and poskf. Then Sentiment score will be calculated.

Once the document is prepared with labels like positive, negative and neutral Naïve Bayes probabilistic Learning approach will executed on it.

\section{(B) Second Objective is to achieve aspect sentiment summarization}

This will be achieved by using Naivy bayes classifer with lexion approach

A naive bayes classifier works by figuring out the probability of different attributes of the data being associated with a certain class. This is based on bayes' theorem. The theorem is $\mathrm{P}(\mathrm{A} \mid \mathrm{B})=\mathrm{P}(\mathrm{B} \mid \mathrm{A}), \mathrm{P}(\mathrm{A}) \mathrm{P}(\mathrm{B}) \mathrm{P}(\mathrm{A} \mid \mathrm{B})=\mathrm{P}(\mathrm{B} \mid \mathrm{A}), \mathrm{P}(\mathrm{A}) \mathrm{P}(\mathrm{B})$. This basically states "the probability of $A$ given that $B$ is true equals the probability of $\mathrm{B}$ given that $\mathrm{A}$ is true times the probability of A being true, divided by the probability of $B$ being true."

\section{A. a). Finding word counts}

We're trying to determine if a data row should be classified as negative or positive. Because of this, we can ignore the denominator. So we have to calculate the probabilities of each classification, and the probabilities of each feature falling into each classification.

We'll then count up how many times each word occurs in the negative reviews, and how many times each word occurs in the positive reviews. This will allow us to eventually compute the probabilities of a new review belonging to each class.

\begin{tabular}{|l|l|l|}
\hline $\begin{array}{l}\text { SentiWordNet 3.0( Name } \\
\text { of product })\end{array}$ & Positive & Negative \\
\hline Iphone 7s & $90 \%$ & $10 \%$ \\
\hline Iphone 6 & $87 \%$ & $13 \%$ \\
\hline
\end{tabular}

Table2: probabilities of a new review

\section{B. b). Making predictions}

Now that we have the word counts, we just have to convert them to probabilities and multiply them out to get the predicted classification. Let's say we wanted to find the probability that the review didn't like it expresses a that sentence is not matched with any of the keywords in negkf

negative sentiment. We would find the total number of times the word didn't occured in the negative reviews, and divide it by the total number of words in the negative reviews to get the probability of $\mathrm{x}$ given $\mathrm{y}$. We would then do the same for $l i k e$ and $i t$. We would multiply all three probabilities, and then multiply by the probability of any document expressing a negative sentiment to get our final probability that the sentence expresses negative sentiment.

We would do the same for positive sentiment, and then whichever probability is greater would be the class that the review is assigned to.

To do all this, we'll need to compute the probabilities of each class occuring in the data, and then make a function to compute the classification.

Algorithm

Step1: determine your train data set

Step2: convert your train data set to frequency data set (frequency table)

Step3: compute prior

$\mathrm{P}(\mathrm{c})=\mathrm{N}_{\mathrm{c}} / \mathrm{N}$

Where $\mathrm{P}(\mathrm{c})$ is the probability of the class

$\mathrm{Nc}$ is the total cost of a particular class in the training set

$\mathrm{N}$ is the total count of class in the training set

Step4: compute the conditional probability/ likelihood of each word attribute

$\mathrm{P}(\mathrm{w} / \mathrm{c})=\operatorname{count}(\mathrm{w}, \mathrm{c})+1 / \operatorname{count}(\mathrm{c})+|\mathrm{v}|$

1. Where $\mathrm{P}(\mathrm{w} / \mathrm{c})$ is the conditional probability /likelihood . where $\mathrm{w}$ is the word attribute and $\mathrm{c}$ is the class

2. Count $\mathrm{w}, \mathrm{c}$ is the total count of word attribute occurs in c class

$3 .+1$ is laplace smoothing

4.Count (c) is the total count of word attribute occurs in a particular class occurs in the training set.

5. $|\mathrm{v}|$ is the vocabulary . count of different word attributes Step5: conditional likelihood probability

Step6: From Step 6, compute posterior probability, it can be calculated by

$$
\left.\mathrm{C}_{\text {map }}=\operatorname{argmax}(\mathrm{P}(\mathrm{x} 1, \mathrm{x} 2, \mathrm{x} 3 \ldots \ldots \mathrm{xn})) \mathrm{P}(\mathrm{c})\right)
$$

\section{FIGURES EXPERIMENTAL RESULTS}

This works also compares the hybrid approach with traditional approaches. i.e, Polarity score is the measure to determine the accuracy of opinion on a different reviews evaluating the performance of proposed approach on several sentiment data sets is as follows.

Table 2: Summary table

$\mathrm{PR}=\mathrm{PWS} / \mathrm{TWS}$
$\mathrm{NR}=\mathrm{NWS} / \mathrm{TWS}$

Sentiment Score $=$ PR-NR

$\mathrm{PR}=$ Positive Ratio, NR=Negative Ratio

$\mathrm{PWS}=$ Positive words in the Sentence

NWS $=$ Negative words in the Sentence 


\begin{tabular}{|l|l|}
\hline Feature & Polarity score \\
\hline Naïve bayes & 88 \\
\hline Hybrid approach & 91 \\
\hline
\end{tabular}

Table3: Comparision between approaches

\section{REFERENCES}

[1] ZiedKechaou, Mohamed Ben Ammar, Adel. M Alimi, "Improving e-learning with sentiment analysis of users' opinions",2011 IEEE Global Engineering Education Conference (EDUCON)

[2] Dan Song, Hongfei Lin, Zhihao Yang, "Opinion Mining in e- Learning System", 2007 IFIP International Conference onNetwork and Parallel Computing - Workshop

[3] N. D. Valakunde, Dr. M. S. Patwardhan,"Multi-Aspect and Multi- Class Based Document Sentiment Analysis of Educational Data Catering Accreditation Process", 2013 International Conference on Cloud \& Ubiquitous Computing \& Emerging technologies.

[4] Stefano Baccianella, Andrea Esuli, and FabrizioSebastiani ,"SENTI WORD NET 3.0: An Enhanced Lexical Resource forSentiment Analysis and Opinion Mining" 2008

[5] Jinan Fiaidhi, Osama Mohammed,Sabah Mohammed Simon Fong, Tai hoon Kim,"Opinion Mining over Twitterspace: Classifying Tweets Programmatically using the R Approach",2013.

[6] Rajdeep Singh, Roshan Bagla, HarkiranKaur,'Text Analytics of Web Posts' Comments Using Sentiment Analysis", 2015.

[7] Bogdan Batrinca Philip C. Treleaven, "Social media analytics: a survey of techniques, tools and platforms", AI \&Soc (2015).

[8] P. Padmavathy and A. Anny Leema "Sentiment Mining from Online Patient Experience using Latent Dirichlet Allocation Method “ in Indian Journal of Science and Technology, Vol 9(19), DOI: 10.17485/ijst/2016/v9i19/93876, May 2016.

[9] C. Scaffidi, K. Bierhoff, E. Chang, M. Felker, H. Ng, C. Jin, Red Opal Produc-

t-featurescoringfromreviews, in:Proceedingsofthe8thACMConference on Electronic Commerce, ACM, 2007, pp.182-191.

Hu,J.Boyd-Graber,B.Satinoff,A.Smith,Interactivetopicmodeling,Mac h. Learn. 95 (3) (2014) 423-469.

[11] Z. Chen, B. Liu, Mining topics in documents: standing on the shoulders of big data, in: Proceedings of the 20th ACM SIGKDD International Conference on Knowledge Discovery and Data Mining, ACM, 2014, pp.1116-1125.

[12] T. Hofmann, Probabilistic latent semantic indexing, in: Proceedings of 22nd

ACMSIGIRConferenceonResearchandDevelopmentinInformationRet rieval, ACM, 1999, pp.50-57.

[13]D.M.Blei,A.Y.Ng,M.I.Jordan,LatentDirichletallocation,J.Mach.Learn. Res.3 (2003)993-1022.

[14]S.Rill,D.Reinel,J.Scheidt,R.Zicari,Politwi:Earlydetectionofemergingpo - litical topics on twitter and the impact on concept-level sentiment analysis, Knowl.-Based Syst. 69 (2014)14-23.

[15] I. Titov, R. McDonald, Modeling online reviews with multi-grain topic mod- els, in: Proceedings of 17th Conference on World Wide Web, ACM, 2008, pp.111-120.

[16]S.Branavan,H.Chen,J.Eisenstein,R.Barzilay,Learningdocument-levelse man-

ticpropertiesfromfree-textannotations,J.Artif.Intell.Res.34(2)(2009)56 9.

[17] H. Wang, Y. Lu, C. Zhai, Latent aspect rating analysis on review text data: a rating regression approach, in: Proceedings of the 16th ACM SIGKDD Inter- national Conference on Knowledge Discovery and Data Mining, ACM, 2010, pp.783-792.

[18]Y.Lu,C.Zhai,N.Sundaresan,Ratedaspectsummarizationofshortcomment $\mathrm{s}$, in:Proceedingsof18thWorldWideWebConference,ACM,2009,pp.131140.

[19] W.X. Zhao, J. Jiang, H. Yan, X. Li, Jointly modeling aspects and opinions with aMaxEnt-LDAhybrid,in:Proceedingsofthe2010ConferenceonEmpirica

\section{AUTHORS PROFILE}

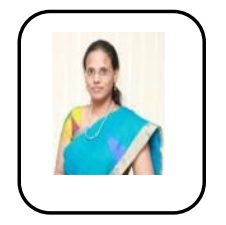

Dr Aruna Kumari Professor at Department of CSE,VJIT, Hyderabad, Telangana India. She is Fellow of CSI( FCSI), and Fellow of IEEE (FIEEE). She is DST Young Scientist Awardee (Govt. of India). She has more than 70 research articles in International Journals and Conferences.

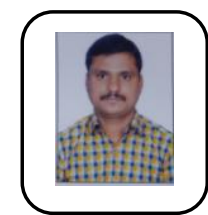

P.Rajashekar, Asst.Professor at Department OF CSE,VJIT,Hyderabad,Telangana India.He is currently pursuing Ph.D. from GITAM university,He has published 5 international journals.

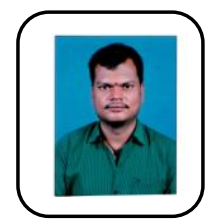

PKVS Sarma,Asst.Professor at Department OF CSE,VJIT,Hyderabad,Telangana India. He has published 4 Papers in international journals 